\title{
Percepción de la primera relación sexual en adolescentes
}

\author{
Perception regarding the first sexual relationship in adolescents
}

\author{
Brenda Sarahí Monter ${ }^{a}$, Eric Hernández López ${ }^{b}$, Verónica Rodríguez Contreras ${ }^{c}$, Sergio \\ Santamaria Suárez ${ }^{d}$, Ulrik Álvarez Martínez ${ }^{e}$, Zarina Isabel Melo Fabela ${ }^{f}$.
}

\begin{abstract}
:
The sexual development of adolescents is implied by physical processes, acceptance of the body image, discovery of sexual needs, personality development, learning about interpersonal relationships, and the establishment of one's own system of sexual values. Therefore, it is important to study the perception of the first sexual relationship, corresponding to the elaboration or expression of a judgement from experience from which beliefs, social norms, power systems and previous meanings regulate the behaviour. The type of investigation is descriptive, with a transversal design in which the main variables correspond to the perception of the first sexual relationship in adolescents, and the associated variables are: V1- cognitive dimension, V2- affective dimension and V3- behaviour dimension. These were analysed according to the following categories: a) the beginning of sex life, b) pregnancy and contraceptive methods, c) sexually transmitted infections and d) social networks. For this, semi-structured interviews were made with five adolescents from the state of Hidalgo who had their first sexual relationship, analysing the intrinsic content vertically and horizontally. In this investigation the behaviour of the first sexual relationship was identified, as well as the influence of social networks. Adolescents are found to be constantly changing their roles and stereotypes concerning their sexuality and sexual practices, and even when they have information, they do not perceive the risks nor preventive behaviour.
\end{abstract}

Keywords:

First sexual relationship, adolescents, perception, cognitive, affective, behavior.

Resumen:

El desarrollo sexual del adolescente está implicado por procesos físicos, aceptación de la imagen corporal, descubrimiento de las necesidades sexuales, desarrollo de la personalidad, aprendizaje de las relaciones interpersonales y establecimiento de un sistema propio de valores sexuales. Por ello es importante estudiar la percepción de la primera relación sexual, correspondiendo a la elaboración o expresión de un juicio a partir de la experiencia, donde influyen creencias, normas sociales, sistemas de poder y significados previos que regulan el comportamiento. El tipo de investigación es descriptivo, de diseño transversal, donde las variables principales corresponden a la percepción de la primera relación sexual en adolescentes, y las variables asociadas son: V1-dimensión cognitiva, V2-dimensión afectiva y V3-dimensión conductual. Estas fueron analizadas de acuerdo a las siguientes categorías: a) comienzo de la vida sexual b) embarazo y métodos anticonceptivos, c) infecciones de transmisión sexual y d) redes sociales. Para ello se llevaron a cabo entrevistas semiestructuradas a cinco adolescentes del estado de Hidalgo, que tuvieron su primera relación sexual, analizando el contenido intrínseco de manera vertical y horizontal. En esta investigación se identifica el comportamiento de la primera relación sexual, así como la influencia de las redes sociales. Los adolescentes se encuentran en constante cambio en sus roles y estereotipos en torno a su sexualidad y prácticas sexuales, aunque tienen información no hay percepción de riesgo, ni conductas preventivas.

Palabras Clave:

a Autor de Correspondencia, Universidad Autónoma del Estado de Hidalgo, https://orcid.org/0000-0002-4185-9386, Email: brenda_islas@uaeh.edu.mx

b Universidad Autónoma del Estado de Hidalgo, https://orcid.org/0000-0002-7052-1683, Email: eric_psicologia@outlook.com c Universidad Autónoma del Estado de Hidalgo, https://orcid.org/0000-0002-7116-8658, Email: veronica_rodriguez5903@uaeh.edu.mx d Universidad Autónoma del Estado de Hidalgo, https://orcid.org/0000-0001-8588-6186, Email: sssergio@uaeh.edu.mx e Universidad Autónoma del Estado de Hidalgo, https://orcid.org/0000-0002-6358-2824, Email: tool-3@ hotmail.com f Universidad Autónoma del Estado de Hidalgo, https://orcid.org/0000-0002-5083-2353, Email: me299710@uaeh.edu.mx 
Primera relación sexual, adolescentes, percepción, cognitivo, afectivo, conductual

\section{Introducción}

La sociedad siempre está en constante cambio, renovando paradigmas, herramientas, tecnología y comportamientos. Los fenómenos que son estudiados en un momento histórico sirven para comprender a la sociedad o a un grupo social de un determinado contexto. La actualización del conocimiento por medio de la investigación sirve para comprender lo que pasa en esta época postmoderna, cuyo propósito es el de desarrollar herramientas psicológicas y de áreas afines que aborden variadas situaciones de una manera eficaz y comprensiva.

La vida cotidiana del ser humano en lo específico parece ser diferente en cada uno, pero en lo general es semejante y predecible. Lo anterior implica la constante observancia del mundo psicosocial en correspondencia de la vinculación dialéctica de las estructuras sociales y sus efectos en las estructuras microsociales, como la familia y los agentes sociales. ${ }^{1}$

Se puede considerar que nuestros contemporáneos tienen una percepción de vivir en una época con cambios drásticos y acelerados en el comportamiento sexual, sin embargo, basta una mirada al pasado para contrastar que las generaciones anteriores hubieran podido extraer la misma conclusión. ${ }^{2}$

Tal es el caso, que en las últimas décadas tenemos sucesos particulares que incluyen ideologías y movimientos sociales como el feminismo y LGBTTTI, que mantienen un discurso en pro al derecho a decidir, sobre la legalización del aborto, la libertad de expresión, un marco jurídico para combatir la discriminación, la libre orientación sexual, el matrimonio homosexual y en general una mayor libertad en la dimisión de la sexualidad que no incluya prejuicios; las nuevas generaciones, principalmente jóvenes se han adoptado de estos movimientos, aumentado la participación y el crecimiento de estos grupos.

"La sexualidad es una de las dimensiones centrales de los seres humanos ya que nacemos como seres sexuales y, desde ese momento los patrones culturales van definiendo comportamientos, significados y actitudes en el transcurso de la vida, por ende, vamos definiendo nuestros gustos y preferencias, descubriendo lo que nos produce placer y deseo." 3,4

Esta dimensión abarca el sexo, las identidades y los roles de género, el erotismo, el placer, la intimidad, la reproducción y la orientación sexual. ${ }^{5}$ "Se vive y se expresa a través de pensamientos, fantasías, deseos, creencias, actitudes, valores, conductas, prácticas, costumbres y relaciones interpersonales. Se puede incluir todos los aspectos mencionados, sin embargo, no todas ellas se vivencian o se expresan siempre." La sexualidad también está influenciada por la interacción de factores biológicos, psicológicos, sociales, económicos, políticos, culturales, éticos, legales, históricos, religiosos y espirituales. ${ }^{6}$

Desde la perspectiva de la psicología, la sexualidad toma relevancia desde las condiciones subjetivas que determinan el desarrollo, así como el estudio de comportamientos saludables y la percepción de riesgo. La psicología abarca tres dimensiones del individuo: 1. Cognitiva; lo que pensamos, 2. Afectiva; lo que sentimos, y 3 . Conductual; lo que hacemos, conformando la triada del comportamiento humano y delimitando su estudio a un aspecto de la vida como la sexualidad o aún más particular como la primera relación sexual. Por otra parte, el desarrollo psicosexual es un proceso complejo, influido por múltiples factores como los genéticos, biológicos, hormonales, neurobiológicos, culturales, sociales y familiares; cada uno de los anteriores estudiados desde las ciencias y disciplinas correspondientes.

Las primeras actitudes hacia el sexo se basan en gran medida en lo que dicen o muestran los padres, amigos y maestros acerca de la sexualidad. "La vertiente psicosocial de la sexualidad se manifiesta desde la colectividad en la que está inmerso el individuo, en cómo se regula la sexualidad mediante normas, presiones parentales y grupos de individuos de la misma edad, con el propósito de inducirnos a encauzar nuestra conducta sexual por una senda determinada, sin perder al individuo como el principal elemento de estudio."6

Luego entonces, la intención de la siguiente investigación es brindar conocimiento de un suceso en específico dentro de la etapa de la adolescencia, la cual es comprendida como aquel periodo del desarrollo humano que se encuentra entre los 10 y 19 años donde se dan importantes cambios físicos, sociales y psicológicos. "Es en esta etapa donde el individuo adquiere una mayor capacidad para elaborar opiniones propias, debatir ideas, defender puntos de vista y formular interrogantes sobre su sexualidad."7,8

Para esta etapa de desarrollo la sexualidad es un conjunto de deseos e inquietudes que se ven influidas principalmente por los medios de comunicación, amigos y padres, que ayudan a formar una identidad, madurez física y emocional que continuamente cambian su vida y la percepción del mundo en el que viven.

Una de las características psicológicas que resultan interesantes durante la adolescencia es el egocentrismo, entendiéndose como un estado de 
ensimismamiento en el cual el adolescente considera que el mundo está centrado en sí mismo, pensándose como protagonista de diversas situaciones sociales. ${ }^{9,10}$ Puede traer percepciones distorsionadas sobre diversas circunstancias, que implican una valoración de determinados sucesos que pueden hacer que se considere especial y más importante en el plano social de lo que son en realidad. Aparecen conductas cuya finalidad es hacerse notar, como considerar que todas las miradas se dirigen a él o ella, por ejemplo, llevar un nuevo corte de cabello o por su complexión. Otra característica peculiar es la invulnerabilidad, en la que piensa que exponerse a cualquier situación de riesgo no implica de ninguna manera un peligro, por ejemplo, el adolescente niega que pueda infectarse, lastimarse o embarazarse, diciendo que al él o ella no le ocurrirá. ${ }^{11}$

\section{La primera relación sexual}

El inicio de la vida sexual es espontánea y no planeada. ${ }^{12,13}$ Ocurre en diferentes grados de intimidad, erotismo y estimulación hasta el coito y el orgasmo. ${ }^{14} \mathrm{La}$ primera relación sexual puede ocurrir por diversos factores como la presión social, los roles de género, el chantaje sentimental, el enamoramiento, la curiosidad o por la simple ausencia de los padres en un día normal. Esta experiencia puede resultar como una de las más significativas en la vida, ya que en ella se lleva a la práctica un conjunto de significados culturales que involucran la relación del propio cuerpo con otro u otros individuos; entre lo desconocido, el miedo, inseguridad, nerviosismo, afecto y relaciones equitativas, también se experimenta placer, aunque en las mujeres generalmente no es así. ${ }^{15,16}$

\section{Embarazo y métodos anticonceptivos}

El embarazo adolescente es considerado un problema de salud pública ya que no es planeado, tiene diferentes puntos de origen como la primera relación sexual, además de que representa un alto riesgo de complicaciones para la adolescente, el feto y el neonato. ${ }^{17}$ "Generalmente suele ser sorpresivo e inesperado ya que es frecuente que los adolescentes lleguen a la relación coital sin utilizar métodos anticonceptivos o la inefectividad de los mismos, así como la falta de administración de métodos de emergencia posterior a la relación coital."15-18, 19,20

\section{Infecciones de Trasmisión Sexual}

Los adolescentes al estar en continua transformación y construcción de su Yo, presentan notorios cambios emocionales, de pensamiento y físicos, los cuales están profundamente relacionados con el inicio de la maduración sexual, pues tienen que ver con el desarrollo de las características psicosexuales, de las cuales depende que puedan surgir prácticas sexuales precoces. Estos cambios los pueden llevar a pensar que no son capaces de contraer alguna Infección de Transmisión Sexual (ITS), que no corren riesgos durante la actividad sexual o por creer que solo ocurre entre personas que tienen relaciones ocasionales. ${ }^{21}$

\section{Redes Sociales}

Actualmente se vive un desarrollo acelerado en las tecnologías de la información y la comunicación, el resultado de esto ha traído un mundo virtual a través de las redes sociales, como Facebook y WhatsApp, entre muchas otras. Su principal función es interactuar con personas que pueden estar a unas cuadras del hogar o en diferentes partes del mundo, podemos comunicarnos con personas que conocemos o desconocidos que posiblemente falsean un perfil o una identidad, también facilitan el recibir y enviar información, desde una simple tarea de la escuela o fotografías con contenido sexual.

"Las redes sociales se han vuelto elementales para estar conectados con el mundo virtual, para los adolescentes no es la excepción ya que han tomado un significado acorde a sus necesidades personales, sexuales y sociales, éstos utilizan las publicaciones y mensajes para remarcan el desarrollo de su identidad, problemas importantes de imagen corporal o para poner en práctica las formas de relacionarse."22

\section{Percepción del inicio de la vida sexual}

Para la psicología la percepción es un proceso que consiste en el reconocimiento, interpretación o valoración de las cosas, a través del procesamiento de información, permitiendo la elaboración de juicios a partir de las sensaciones captadas del medio ambiente. ${ }^{23}$

Se ha retomado a Merandi y Ricoeur para seleccionar elementos teóricos desde sus campos de estudio permitiéndonos construir el concepto desde una mirada psicosociocultural, de tal manera que la percepción la definimos como: un proceso por el cual los órganos de los sentidos captan el ambiente, configurando la información sensorial mediante estilos lingüísticos y culturales, permitiendo objetivar y describir un sentido particular de la realidad. ${ }^{24,25}$

Por lo que, la percepción de la primera relación sexual corresponde a la elaboración o expresión de un juicio a partir de la experiencia, de sensaciones captadas por el cuerpo, así como de creencias, normas sociales y significados previos que influyen en el comportamiento. 
Desde otra perspectiva, la primera relación sexual constituye un elemento fundamental en el desarrollo humano, en el que hay contacto físico entre personas y que puede tener como objetivo, dar o recibir placer sexual, o con fines reproductivos. ${ }^{17}$ Según sea la percepción de la relación sexual del adolescente, se describirá como puede ser, por ejemplo, agradable o desagradable, así como integrar características como doloroso, sudoroso, rápido, bonito, malo, bueno, excelente, grande, pequeño, rugoso, suave, oloroso, etcétera.

Es el objetivo de este trabajo analizar la dimensión cognitiva, afectiva y conductual de los adolescentes en relación a la percepción de su primera relación sexual, permitiendo una comprensión de cómo viven su sexualidad y cómo esta repercute en su vida, considerando los criterios de planeación, emociones, presión social, embarazo, métodos anticonceptivos e infecciones de trasmisión sexual.

\section{Método}

El tipo de investigación es descriptivo, de diseño transversal. ${ }^{26}$ Las variables principales corresponden a la percepción de la primera relación sexual en adolescentes, las variables asociadas son: V1-dimensión cognitiva, V2dimensión afectiva y V3-dimensión conductual. Estas fueron analizadas de acuerdo a las siguientes categorías: a) comienzo de la vida sexual, b) embarazo y métodos anticonceptivos, c) infecciones de transmisión sexual y d) redes sociales.

Preguntas de investigación enfocadas a la muestra:

- ¿Cuál es la percepción que tienen los adolescentes en su primera relación sexual?

- ¿Cuáles son las emociones en la experiencia de la primera relación sexual?

- ¿Cuáles son los pensamientos en la experiencia de la primera relación sexual?

- ¿Cuáles son las conductas en la experiencia de la primera relación sexual?

La muestra está conformada por cinco adolescentes, hombres y mujeres de entre 16 a 19 años de edad, residentes del estado de Hidalgo, México.
Tabla 1. Datos generales de los participantes del estudio

\begin{tabular}{ccccc}
\hline Informantes & Edad & Sexo & $\begin{array}{c}\text { Orientación } \\
\text { sexual }\end{array}$ & $\begin{array}{c}\text { Edad } \\
\text { (primera } \\
\text { relación } \\
\text { sexual) }\end{array}$ \\
\hline E1 & 16 & Mujer & Heterosexual & 14 \\
E2 & 16 & Mujer & Homosexual & 14 \\
E3 & 17 & Hombre & Heterosexual & 16 \\
E4 & 18 & Mujer & Heterosexual & 15 \\
E5 & 19 & Mujer & Heterosexual & 15 \\
\hline
\end{tabular}

Criterios de inclusión:

- Adolescentes del Estado de Hidalgo, hombres y mujeres que hayan tenido su primera relación sexual.

- Que acepten participar como informantes.

- Que firmen el consentimiento informado.

- Que completen la entrevista.

Se utilizó como instrumento de medición, la entrevista semiestructurada, directa (de acuerdo a las categorías).

Procedimiento:

1) Contactar adolescentes con las características requeridas del estudio.

2) Informar sobre el objetivo del estudio, las características de la muestra y la confidencialidad.

3) Identificar a las posibles personas participantes en la investigación.

4) Contactar indirectamente con el adolescente, por medio de la persona que nos informó o contactar directamente invitando a ser participante.

5) Se formaliza el día, la hora y el lugar de la entrevista; se cubren gastos de trasporte.

6) Solicitar la firma del consentimiento informado.

7) Aplicación de entrevista.

8) Elaboración de transcripción de entrevistas por ejes temáticos.

9) Se realizó un análisis de la información intrínseca, misma que permite responder las preguntas y los objetivos de investigación. ${ }^{27}$

10) Redacción de resultados y conclusiones.

\section{Resultados y discusión}

A los adolescentes se les da un valor socialmente como inexpertos y demasiado jóvenes para el sexo. A pesar de que muchos hayan alcanzado madurez biológica para la reproducción, no lo han hecho en la dimensión psicológica 
y social para enfrentar todos los riesgos y consecuencias que puede traer la primera relación sexual. ${ }^{28}$

Comienzo de la vida sexual: planeación, emociones y presión social. El inicio de la vida sexual es espontáneo, tres de las personas entrevistadas, no planearon su primera relación sexual, mientras que las otras dos personas hablaron previamente sobre la relación sexual y la planeación. ${ }^{12}$

Se supone que fuimos a hacer un trabajo, pero sólo estábamos dos amigos y mi novia... ellos estaban en la sala y nosotras en el cuarto. (E2)

La oportunidad de tener relaciones sexuales se llega a presentar cuando los adultos están ausentes, en salidas espontaneas, en un evento no planeado o en la casa de alguno de los integrantes de la pareja. ${ }^{29}$

Tres de los adolescentes buscaron lugares expuestos y en apariencia aislados para tener su primera relación sexual: Era un terreno totalmente baldío... eran como las 11 de la noche... me llevó a un árbol grande que le caigan las ramas y hacía frío. (E1)

Por otra parte, la planeación la dirige principalmente el hombre de forma sutil y persistente, generalmente utilizando o mostrando afecto. ${ }^{15}$ En el caso de las mujeres, se sintieron confundidas sobre sus sentimientos hacia su pareja, ya que es frecuente el chantaje sentimental por parte de los hombres para acceder a una relación sexual que aparenta el consentimiento: Yo sí sentí maripositas en el estómago cuando él me decía, es que me gustas y quiero que tu primera vez sea conmigo. Yo sí sentía algo por él, y sí estaba dispuesta a sentir más, pero pues no, ya no se dio. (E1)

Existen factores psicológicos positivos en la primera relación sexual como la satisfacción, el orgullo, tranquilidad y el sentimiento de realización, mientras, que en los negativos se encuentra la culpa, tristeza, arrepentimiento, vergüenza y preocupación. $^{12}$ Cuatro participantes indican que sintieron emociones negativas cómo las que indica el autor, por ejemplo, nerviosismo, zozobra, miedo, vergüenza y culpa: Nerviosismo, no sabía qué hacer... no sabía si la podía lastimar si le iba a gustar... más que nada fueron muchos nervios. (E2)

La presión social puede ocurrir principalmente por los pares, en el caso de los hombres adolescentes, suelen hablar sobre sus experiencias sexuales con el objetivo de sentirse parte de ese grupo o mantener cierto estatus, para no ser considerado anormal o un caso raro. ${ }^{15}$ En contraparte, para las mujeres puede resultar diferente debido a que la presión es menor, empero, en esta investigación se encontró que entre adolescentes mujeres también se percibe un estatus: Eran así, como pláticas para contarnos su experiencia A lo mejor, así como para sentirse más grandes o importantes ante nosotros. (E1)
Las adolescentes también consideraban ser de las primeras de su grupo de amigos que experimentaron su primera relación sexual y lo platicaban abiertamente con amigas y compañeras de escuela. Estos factores tienen la cualidad para ejercer presión en sus amigas o para ser fuente de información y curiosidad: Creo que yo fui de las primeras, tenía una amiga en primero que me contaba cosas porque era mayor. La acompañaba a comprar pruebas de embarazo, condones; una vez la acompañe por una pastilla del día siguiente. (E4)

Infecciones de transmisión sexual:

Las ITS se presentan frecuentemente en aquellos individuos que mantienen conductas y actitudes sexuales riesgosas, entre ellas tener más de una pareja sexual, practicar coito de forma no segura o no usar preservativo.

Los adolescentes pueden llegar a pensar que no son capaces de contraer alguna Infección de Transmisión Sexual o que no corren riesgos durante la actividad sexual. 21 De acuerdo a esto los resultados concuerdan, ya que ninguno de los entrevistados consideró que se pudiera infectar o su percepción de riesgo era incompetente: Confiaba en que no tenía ninguna enfermedad, me hubiera comentado y hubiéramos usado algún método anticonceptivo como el condón. (E3)

Embarazo y métodos anticonceptivos:

"La prevalencia del embarazo adolescente ha aumentado considerablemente lo que se ha transformado en un problema de salud pública." 30 Dos entrevistadas indicaron que es muy común ver a sus amigas embarazadas con problemas físicos y psicológicos, observan que sufren mucho en ámbitos escolares, sociales y familiares: estábamos en segundo de prepa y ya muchas de mis compañeras salían embarazadas... dejaron de hacer muchas cosas dejaron de salir, se mataban haciendo cosas como trabajar o hacer tareas muy tarde y para mí también era muy triste. (E5)

Las adolescentes identifican el miedo por ver a sus compañeras o amigas embarazadas, ya que coartan sus proyectos de vida, afectan su calidad de vida, hay un cambio de hábitos y aumenta la probabilidad de abandono escolar. ${ }^{18}$

La falta de información sobre el proceso del embarazo y determinadas creencias ocurren por factores como los tabúes, por cómo se lleva la educación sexual en las escuelas, en casa y entre amigos, estos últimos suelen ser los que informan o brindan conocimiento que puede resultar falso o desorientador: Antes habíamos tenido como un faje, yo pensé ¿Por qué no me bajaba? Tuve diez días de retraso, entonces yo dije estoy embarazada. (E4) 
No hacer uso de anticoncepción durante la relación sexual se asocia con el desconocimiento sobre la efectividad de los métodos anticonceptivos, señalando que los adolescentes tienen poco o nulo conocimiento sobre estos, sin embargo, los jóvenes que perciben los beneficios del condón son más propensos a utilizarlo en sus relaciones sexuales." ${ }^{11}$ Tres de los entrevistados indican que no usaron ningún método anticonceptivo en su primera relación sexual y esos mismos señalan que no tenían información o desconocían de cómo se usaban: No, no pensé usar condón en ese momento... un día después me llevó unas pastillas, creí que las pastillas del día siguiente eran difíciles de conseguir... sabía que detenía los espermatozoides o cerraba algo, pero realmente no sabía que reacciones tiene. (E1)

Esto no cambia en las relaciones homosexuales, ya que la entrevistada dos, refiere que, aunque sabía que no se puede embarazar, si puede contraer alguna infección, sin embargo, no sabía de métodos anticonceptivos para tener una relación de mujer con mujer, indicando que después en internet conoció como usar un condón: No se me vino a la cabeza o sea a pesar de que entre lesbianas no podemos utilizar para el embarazo, si para las enfermedades o infecciones. (E2)

A partir de que los entrevistados tuvieron su primera relación sexual comenzaron a buscar información sobre los métodos anticonceptivos, esto modifica sus pensamientos y significados previos. También indica quienes son los que tienen menor o mayor probabilidad de utilizar un método en posteriores relaciones sexuales ya que utilizar el condón en la primera relación tiene mayor probabilidad de usarlo en sus posteriores relaciones sexuales, reforzando la conducta saludable, la información y una percepción de riesgo adecuada. ${ }^{31}$

Redes sociales:

La red informática permite tener mayor indagación sobre la sexualidad, principalmente en las redes sociales y páginas pornográficas, que transforman la visión y la forma de ejercer la actividad sexual en los adolescentes. ${ }^{32}$ Los entrevistados perciben que el medio menos popular en esta época es la televisión a comparación de las redes sociales; que algunos programas muestran contenido sexual y otras pretenden tener la función de educación sexual, sin embargo, influye que se arriesguen más ya que la información que se transmite en ese medio no incluye un contenido preventivo: Yo nunca he visto antes algún comercial sobre alguna prevención hasta ahora, los he visto más en Facebook. (E5)

La mayoría de los entrevistados concuerdan que las redes sociales se han vuelto un medio que proporciona información en temas de sexualidad, por lo que se arriesgan a ver información poco fundamentada, lo que puede producir que tengan conductas de riesgo en su primera relación sexual. ${ }^{32}$ La red social que más indican para obtener temas de educación sexual es Facebook y YouTube: una de mis amigas me decía, pues si quieres hacerlo bien entra a Google para que entiendas o en YouTube. (E1)

Por otra parte, los adolescentes buscan personas en las redes sociales o internet, con un objetivo afectivosexual. ${ }^{33} \mathrm{La}$ entrevistada número uno relata que su primera relación sexual fue con alguien que conoció en Facebook y con el que tuvo la intención de tener una relación afectiva, pero lo que obtuvo fue sólo tener su primera relación sexual desde la primera vez que lo conoció en persona: Por las redes sociales fue que conseguí tener mi primera vez...yo si sentía algo por él y estaba dispuesta a sentir más por él y pues no, ya no dio. (E1)

La práctica del sexting forma parte de la vida cotidiana, está naturalizada y es popular en los diferentes grupos de adolescentes. ${ }^{33}$ Las herramientas predilectas para el intercambio de imágenes y videos con fines eróticos y masturbatorios son Snapchat y WhatsApp. Sin embargo, el concepto de sexting no ha permeado en la realidad local y para hacer referencia a los intercambios sexuales virtuales, los adolescentes utilizan la palabra de origen anglosajón pack.

Tal como lo alude una de las entrevistadas, la red social WhatsApp es una de las herramientas más accesibles para compartir videos o imágenes explicitas. Otras entrevistadas señalan que es una práctica común y que el único requisito es tener cautela y confianza a las personas que se lo comparten. Tres de las entrevistadas refieren que sus padres o familiares han visto sus fotos intimas, ya sea por un error de envió o por filtración de la información, por lo que se puede inferir que es un riesgo y vulnera a la persona por la información que es publicada o enviada por las redes sociales. Son estas las nuevas problemáticas a las que se enfrentan los adolescentes al mostrar su sexualidad por las redes sociales: Entonces, yo sí lo hacía... he mandado fotos semidesnudas, pero después esas fotos llegaron a las manos de mi mamá y mi papá. (E1)

\section{Conclusiones}

Los acontecimientos sociales y políticos en los últimos años han transformado la conducta, afectos y pensamientos en la adolescencia, por ello es destacable la actualización y revisión de las nuevas prácticas sexuales que surgen durante el periodo transitorio de la adolescencia y adultez, que pueden determinar el comportamiento en relación a diversos factores que influyen en general a la sociedad, como pueden ser 
embarazos no planeados o infecciones de transmisión sexual.

Dentro de la investigación, en el desarrollo sexual del adolescente participan factores como el proceso físico, la aceptación de la imagen corporal, el descubrimiento de las necesidades sexuales, el desarrollo de la personalidad, el aprendizaje de las relaciones sexuales y el establecimiento de un sistema propio de valores sexuales, todo ello mientras el adolescente está sometido a la presión ejercida por su grupo de iguales o a las reacciones de sus padres ante ese desarrollo sexual.

Para los participantes la primera relación sexual en la adolescencia generalmente se embellece como algo trascendente, con lo cual termina siendo objeto de deseo, culpa y ambivalencia, aunque puede resultar como un ejercicio de poder que impone alguna de las dos personas, por lo que, puede ser un evento no placentero, con múltiples riesgos y considerado socialmente como malo.

La primera relación sexual de los adolescentes no suele tener una planeación concreta, puede ser en casas de familiares o cuando hay ausencia de figuras de autoridad cómo los padres, también puede suceder en lugares accesibles y expuestos a ser descubiertos, además la planeación está organizada por la persona que tenga mayor edad. Se experimenta miedo y nerviosismo, así como afecto principalmente por las mujeres, esto también se refleja en la preocupación por la satisfacción de su pareja, incluso tomando un rol dominante intentado demostrar que se tiene la experiencia.

El embarazo para los adolescentes es un tema preocupante, ya que cuando tienen relaciones sexuales mencionan que tienen temor, pero eso no significa que usen algún método anticonceptivo para prevenirlo.

Respecto a las infecciones de transmisión sexual, a pesar de tener información sobre el tema, no tienen una percepción de riesgo adecuada, en el caso de los adolescentes se puede deber a la confianza que le tienen a su pareja a pesar de tener poco tiempo de conocerlos. Hay mayor preocupación por un embarazo que por una ITS, además de tener creencias e información errónea sobre el embarazo, es poco probable que usen el condón y es la persona con mayor edad quien generalmente toma la decisión. Desconocen el uso correcto del condón y de otros métodos anticonceptivos, es hasta que tienen su primera relación sexual que se comienzan a informar y a tener práctica para colocar el condón. Además, el momento de la primera relación suele ser rápida con nerviosismo y miedo, centran toda su atención en el momento, las emociones y las sensaciones del cuerpo, por lo que pueden olvidar cualquier tipo de conductas preventivas.
Por otra parte, los medios de comunicación y las tecnologías de la información han resultado ser un elemento fundamental para conocer prácticas y formas de relacionarse previo a la primera relación sexual, así como la difusión de información privada. Dentro de las redes sociales populares esta WhatsApp, que es utilizado principalmente para difundir packs en chats grupales, sin importar perder la privacidad y vulnerando a la persona por la información difundida, en cuanto a Facebook, es ocupado para mantener conversaciones con personas desconocidas, adultas o que conocen a distancia, pero que nunca les han hablado en persona. La comunicación con mensajes sexuales es muy común en las redes sociales de los adolescentes, de alguna manera se ha normalizado y no se percibe como algo malo, sin embargo, los descuidos con la información son muy comunes, ya que su información suele llegar a familiares o es enviada por accidente a chats familiares. Respecto a los programas de televisión son considerados como exagerados y que no proporcionan información adecuada o suficiente, incluso son las redes sociales como Facebook y YouTube, de donde suponen que pueden obtener información veraz.

Actualmente las redes sociales se han vuelto un medio para relacionarse con personas desconocidas, lo cual representa un riesgo que puede no ser identificado por el adolescente. En los últimos años se han desarrollado nuevos comportamientos de índole sexual que realizan los adolescentes a través de las redes sociales. 34 Hoy en día, los fenómenos que se presentan con mayor frecuencia son el sexting, el cibersexo y las relaciones afectivo-sexuales virtuales.

Esta investigación permitió observar y describir cómo son las relaciones interpersonales de padres, maestros y amigos con la muestra estudiada, por lo que en ausencia de comunicación afectiva e informada sobre temas relacionados con la primera relación sexual conlleva a que se puedan realizar prácticas consideras como riesgosas. El tema de la sexualidad durante la adolescencia inaugura un comportamiento inquisitivo, llevándolo posiblemente a experimentar primeramente con su cuerpo y posteriormente con alguien que compartirá intimidad sexual. Para el adolescente puede tomar diferentes significados o emociones, sin embargo, esta experiencia será muy valiosa dentro de su desarrollo humano, por lo que, es indispensable la orientación y comunicación que puedan tener en el hogar, ya que la familia juega un papel de suma importancia, así como a la educación que puedan recibir en el ámbito escolar.

\section{Referencias}

[1] Mora C. La observación dialéctica: problemas de método en investigaciones educativas [Internet]. Buenos Aires: CLACSO, Consejo Latinoamericano de Ciencias Sociales; 2001. Disponible en: 
http://bibliotecavirtual.clacso.org.ar/clacso/gt/20101010032719/7ninci .pdf

[2] Blair E. Aproximación teórica al concepto de violencia: avatares de una definición [Internet]. Colombia: Scielo; 2009. Disponible en http://www.scielo.org.mx/pdf/polcul/n32/n32a2.pdf

[3] Freud S. Introducción al psicoanálisis. 2ª ed. México: Alianza; 1992.

[4] García M., \& Godino M. Hablemos sobre sexualidad. Revista Universidad Nacional Politécnica de Rosario. 2019; 31 (01):1-5.

[5] Secretaria de Salud del Distrito Federal. Por mí, por ti, por tod@s información vital para la salud sexual. 3. ${ }^{a}$ ed. México ; 2015.

[6] Organización Mundial de la Salud. Defining sexual health Report of a technical consultation on sexual health. Geneva: Asociación Mundial de Sexologia; 2006. Disponible en: https://www.who.int/reproductivehealth/publications/sexual_health/de fining_sexual_health.pdf

[7] Organización Mundial de la Salud. Desarrollo en la adolescencia. 2018. Disponible https://www.who.int/maternal_child_adolescent/topics/adolesc ence/dev/es/

[8] Papalia DE., \& Martorrell G. Desarrollo humano. México: Mc Graw Hill. 2017

[9] Mckinney JP., Fitzgerald H., \& Strommen E. Psicología del desarrollo en edad adolescente. México: El Manual Moderno. 1982.

[10] Feldman, RS. Desarrollo psicológico a través de la vida. México: Pearson Educación. 2007

[11] Santrock J. Psicología del desarrollo, el ciclo vital. Madrid, España: McGraw Hill. 2006

[12] Vargas E., \& Barrera F. Adolescencia, relaciones románticas y actividad sexual: una revisión. Revista Colombiana de Psicología. 2002; 11: 115-134. Disponible en: http://www.redalyc.org/articulo.oa?id=80401109

[13] Vargas Trujillo E., \& Barrera F. Relaciones Padres-Hijos y su asociación con las relaciones románticas y sexuales de los adolescentes. (Informe de Investigación). Bogotá, Colombia: Universidad de Los Andes, Departamento de Psicología. 2002.

[14] Denmark F., Rabinovittz V., \& Sechzer J. Engendering Psychology. USA: Allyn and Bacon. 2000

[15] Welti C. Inicio de la vida sexual y reproductiva. Pap. Poblac. 2005; 11 (45). Disponible en http://www.scielo.org.mx/scielo.php?script=sci_arttext\&pid=S140574252005000300007

[16] Rincón-Silva EA. Primera relación sexual en adolescentes escolares de Montelíbano (Córdoba, Colombia): un análisis desde sus relatos. Revista Colombiana de Enfermería. 2013; 8: 61-73. Disponible en: https://www.researchgate.net/publication/327352034_Primera_relacio n_sexual_en_adolescentes_escolares_de_Montelibano_Cordoba_Colo mbia_un_analisis_desde_sus_relatos

[17] Mendoza LA., Claros DI., \& Peñaranda CB. Actividad sexual temprana y embarazo en la adolescencia: estado del arte. Chil obstet ginecol. 2016;81 (3): $243-253 . \quad$ Disponible en https://scielo.conicyt.cl/scielo.php?script=sci_arttext\&pid=S071775262016000300012 .

[18] Cogollo R. Aspectos biopsicosociales asociados al embarazo adolescente. Cuidarte. 2012; 3 (1): 385-393. Disponible en: http://www.redalyc.org/articulo.oa?id=359533179019

[19] Organización Mundial de la Salud. El embarazo en la adolescencia 2019. Disponible en: https://www.who.int/es/news-room/factsheets/detail/adolescent-pregnancy

[20] García M., Silva ME., Ortiz M, Pulliquitín T., \& Pavón MF. Embarazo precoz, determinación factores y consecuencias psicosociales. Ecuador: UCE. 2016.
[21] Castro I. Conocimientos y factores de riesgo sobre infecciones de transmisión sexual en adolescentes. Revista Habanera de Ciencias Médicas. 2010; 9(3): 705-716.

[22] Peris M., Maganto C., \& Kortabarria L. Autoestima corporal, publicaciones virtuales en las redes sociales y sexualidad en adolescentes. European Journal of investigation in health, psychology and education. España. 2015; 3(2): 171-180. Disponible en: https://formacionasunivep.com/ejihpe/index.php/journal/article/view/ $39 / 26$

[23] Red D. Hacia una concepción integrada de la mente. Revista Venezolana de Información, Tecnología y Conocimiento. 2010; 7 (1): 123-136. Disponible en: https://www.redalyc.org/articulo.oa?id=823/82312576008

[24] Merani A. Diccionario de psicología. Barcelona, España: Grijalbo. 1997

[25] Ricoeur P. Freud: una interpretación de la cultura. México: Siglo XXI. 1985

[26] Hernández-Sampieri R, Fernández-Collado C y Baptista-Lucio P. Metodología de la Investigación. 6 ed. México: McGraw-Hill. 2014

[27] Hernández E., \& Islas BS. Percepción simbólica de la primera relación sexual en adolescentes [Licenciado]. Universidad Autónoma del Estado de Hidalgo; 2019.

[28] Lara Ortega F., \& Heras Sevilla D. Formación sobre sexualidad en la primera etapa de la adolescencia. Datos obtenidos en una muestra de $2^{\circ}$ y $3^{\circ}$ de eso en burgos. International Journal of Developmental and Educational Psychology. 2008; 1(1): 241-248.

[29] Harvey J., Wenzel A., \& Sprecher S. The Handbook of Sexuality in Cióse Relationships. New Jersey: Lawrence Erlbaum Associates Publishers. 2004

[30] Márquez Á., Verdecia C., \& Herrera V. Embarazo y adolescencia: factores biológicos materno y perinatal más frecuentes. Revista Cubana Obstet Ginecol. 2001; 27(2): 158-64

[31] Villalobos A., De Castro F., Rojas R., \& Allen B. Anticoncepción en adolescentes mexicanos de escuelas del nivel medio superior: uso y necesidades insatisfechas. 20017; 59 (5). Disponible en: http://www.scielo.org.mx/scielo.php?script=sci_arttext\&pid=S003636342017000500566\#aff1

[32] Giraldo León C. Cibercuerpos: los jóvenes y sexualidad en la posmodernidad. Revista Electrónica "Actualidades Investigativas en Educación". 2013; 13(1): 1-22. Disponible en: http://www.redalyc.org/articulo.oa?id=44725654006

[33] Gelpi G., Pascoll N., \& Egorov D. Sexualidad y redes sociales online: Una experiencia educativa con adolescentes de Montevideo. Revista Iberoamericana de Educación. 2019; 80(2): 61-80.

[34] Arab L., \& Díaz G. Impacto de las redes sociales e internet en la adolescencia: aspectos positivos y negativos. Revista Médica Clínica Las Condes. 2015; 26(1): 7-13. 